UDC 533.9

\title{
Space-charge wave in a dusty plasma column containing collisional streaming ions
}

\author{
Myoung-Jae Lee ${ }^{1 *}$, Young-Dae Jung ${ }^{2}$ \\ ${ }^{1}$ Department of Physics, Hanyang University, Seoul 04763, South Korea \\ ${ }^{2}$ Department of Applied Physics and Department of Bionanotechnology, \\ Hanyang University, Ansan, Kyunggi-Do 15588, South Korea \\ "e-mail: ydjung@hanyang.ac.kr
}

\begin{abstract}
In this work, we have obtained the dispersion relation for the space-charge wave propagating in a dusty plasma column containing collisional streaming ions by using the fluid equations and the Poisson equation which lead to a Bessel equation. The growth rate of the space-charge and the geometric and collisional effects on the growth rate have been investigated. It is shown that the space-charge wave can be excited for a large axial wave number. The growth rate of excitation increases as the order of the roots of the Bessel function increases. It is also found that the growth rate decreases with an increase of the radius of column as well as with an increase of the collision frequency. In addition, it is found that the disturbance of wave can be damped only for small wave numbers and the ion collision plays a significant role in the physical properties of the space-charge wave.
\end{abstract}

Key words: Space-charge wave; Dust plasma

PACS: $36.40 . \mathrm{Gk}$

\section{Introduction}

The effects of radial boundaries of plasma columns on plasma waves have been investigated previously [1-5]. A decade ago, the behavior that the radial boundary leads to a frequency cutoff in the dust-acoustic wave dispersion relation was reported $[6,7]$. Recently, the plasma waves in a radially bounded system such as a column plasma were reported to account for the Landau damping of dustacoustic space-charge waves, diffusion effects on the dissipation of waves in a turbulent plasma column, wake potential effects on the propagation of dust ion-acoustic waves, etc. [8-10]. The dispersion relations for plasma wave in a radially bounded collisional dusty plasma also have drawn much interests since they explain the boundary effects for the streaming charged particles in an electric field and the dissipative ion-dust streaming instability [3, 11]. The streaming instability plays an important role in various plasmas and astrophysical environments since it is connected to various physical dynamic phenomena including the heating mechanism in plasma systems [12-17]. For instances, the effects of non-Maxwellian ion-dust streaming instability, the effects of cyclotron motion in a positron-electron pair plasma, dust-dust two streaming instability in dust clouds, etc. have been recently reported [18-20]. In this work, we are motivated to study the excitation and dissipation of the plasma waves in a column dusty plasma containing the collision-dominated streaming ions. To the best of our knowledge, the boundary effects on the excitation and dissipation of the plasma wave in a collisional two-stream dusty plasma in a column has not been reported yet. Since the ion stream is collision dominated, the susceptibility of the ion must contain the appropriate collision frequency [13, 21]. Then the fluid equations for each charged particle species and the Poisson's equation will construct a closed system to yield a Bessel equation whose solution is finite at the origin and null at the boundary of the column dusty plasma. Then, we obtain the new results on the dispersion relation for the space-charge wave propagating in a column dusty plasma containing collision-dominated ion flow. The growth rate of the space-charge wave is also derived and the geometric and collisional effects on the growth rate are investigated.

\section{Numerical Simulation}

Our duty plasma is uniform, bounded by a column, and contains electrons, singly charged ions, and negatively charged dust grains of the charge state $Z_{d}$ so that the dust charge is denoted as $q_{d}=-Z_{d}$. In the equilibrium, the quasineutrality condition yields, $n_{e 0}+Z_{d} n_{d 0}-n_{i 0}=0$, where $n_{j 0}$ is the equilibrium density of the plasma species $j(=e$, $I, d$ for electrons, ions, and dust grains, 
respectively). The plasma has a cylindrical geometry with coordinates $(r, \theta, z)$ and is azimuthally symmetric so that there is no $\theta$-dependence. We shall consider that the curl of electric field will be vanished, i.e., the perturbation is electrostatic. Under these conditions, plasma motions on the $r-\theta$ plane can be ignored and the wave propagation in the $z$-axis is important. The system is then described using the continuity, momentum, and Poisson's equations for each charged particle species given by

$$
\begin{gathered}
\frac{\partial n_{j}}{\partial t}+\nabla \cdot\left(n_{j} \mathbf{v}_{j}\right)=0 \\
m_{j} n_{j}\left(\frac{\partial \mathbf{v}_{j}}{\partial t}+\mathbf{v}_{j} \cdot \nabla \mathbf{v}_{j}\right)=-\nabla P_{j}-q_{j} n_{j} \nabla \varphi
\end{gathered}
$$

and $\nabla^{2} \varphi=-4 \pi \sum_{j=e, i, d} q_{j} n_{j}$, respectively. Here, the symbols $n_{j}, v_{j}, P_{j}, q_{j}$, and $\varphi$ denote the density, velocity, pressure, and electrostatic potential, respectively. We linearize the above equations by letting $n_{j}=n_{j 0}+n_{j 1}, v_{j}=v_{j 0}+v_{j 1}$, and $\varphi=\varphi_{1}$ where the quantity with subscript 0 denotes the equilibrium quantity and the quantity with subscript 1 denotes the small deviation from its equilibrium values. We let $\varphi_{0}=0$. In a cylindrical coordinate system, the perturbed quantities $n_{j 1}, v_{j 1}$, and $\varphi_{1}$ are assumed to vary as $[22,23]$ :

$$
\begin{aligned}
n_{j 1}(\mathbf{r}, t) & =\bar{n}_{j 1}(r) e^{i\left(k_{z} z-\omega t\right)-i \xi \theta}, \\
\mathbf{v}_{j 1}(\mathbf{r}, t) & =\overline{\mathbf{v}}_{j 1}(r) e^{i\left(k_{z} z-\omega t\right)-i \xi \theta}, \\
\varphi_{1}(\mathbf{r}, t) & =\bar{\varphi}_{1}(r) e^{i\left(k_{z} z-\omega t\right)-i \xi \theta},
\end{aligned}
$$

where $\bar{n}_{j 1}(r), \overline{\mathbf{v}}_{j 1}(r)$, and $\bar{\varphi}(r)$ are the amplitudes of perturbation in the transverse direction and $k_{z}$ is the propagation wave number along the axial $z$ direction of the column, $k_{r}$ is the transverse wave number, and $\xi$ is the separation constant for the azimuthal angle $\theta$. For the azimuthally symmetric system, we have the following form of the differential equation for the transverse potential $\bar{\varphi}_{1}(r)$ [23] by linearizing Eqs. (1) and (2) with Poisson's equation,

$$
\frac{d^{2} \bar{\varphi}_{1}(r)}{d r^{2}}+\frac{1}{r} \frac{d \bar{\varphi}_{1}(r)}{d r}+\beta^{2} \bar{\varphi}_{1}(r)=0,
$$

where the separation parameter $\beta^{2}$ is given by the plasma dielectric function $\varepsilon_{l}\left(\omega, k_{z}\right)$ in the form $\beta^{2} / k_{z}^{2}=-\varepsilon_{\ell}\left(\omega, k_{z}\right)$. In Eq. (6), the solution can be written as $\bar{\varphi}_{1}(r)=d_{1} J_{0}(\beta r)+d_{2} N_{0}(\beta r)$ where $J_{0}(\beta r)$ is the zeroth-order Bessel function of the first kind and $N_{0}(\beta r)$ is the zeroth-order Neumann function, with constant coefficients $d_{1}$ and $d_{2}$. The boundary condition at $r=R$ requires that the transverse potential must be zero, i.e., $\bar{\varphi}_{1}(R)=0$, where $R$ is the radius of the column. At the origin, we must have finite potential, i.e., $c_{2}=0$. Therefore, at $r=R$, we just have $J_{0}(\beta R)=0$ and the separation parameter $\beta$ is determined by $\beta R=\alpha_{0 n}$, where $\alpha_{0 n}$ are the $n$ th-roots of $J_{0}(\beta R)=0$, i.e, $\alpha_{01}=$ $2.4048, \alpha_{02}=5.5201, \alpha_{03}=8.6537$, etc. We now consider a cold ion stream in a cold dusty plasma where the streaming ions are collision dominated, whereas the electrons and dusty particles are stationary. Here, we assume that the collision dominant ions are flowing in the $z$-direction. The longitudinal dielectric permittivity for a cold plasma with streaming ion reads [13]:

$$
\begin{gathered}
\varepsilon_{\ell}\left(\omega, k_{z}\right)=1-\omega_{p e}^{2} / \omega^{2}+ \\
+i \omega_{p i}^{2} /\left[v\left(\omega-k_{z} u_{i}\right)\right]-\omega_{p d}^{2} / \omega^{2}
\end{gathered}
$$

where $\omega_{p j}=\left(4 \pi n_{j} q_{j}^{2} / m_{j}\right)^{1 / 2}$ is the plasma frequency of species $j$. After some mathematical manipulations, we obtain

$$
\begin{gathered}
\omega\left(k_{z}\right) \approx \omega_{p e}\left(1-\frac{\alpha_{0 n}^{2}}{k_{z}^{2} R^{2}}\right)^{1 / 2}- \\
-\frac{i \omega_{p i}^{2} \omega_{p e}}{2 v\left(\omega_{p e}-k_{z} u\right)}\left(1-\frac{\alpha_{0 n}^{2}}{k_{z}^{2} R^{2}}\right)^{-1 / 2},
\end{gathered}
$$

where we assume for simplicity $\omega \approx \omega_{p e}$ and $\omega>>\omega_{p d}$ provided that $\alpha_{0 n} / k_{z} R<1$. Here, for $k_{z}>\omega_{p e} / u_{i}$, the instability of the wave occurs. Therefore, the system can be unstable in spite of the collisional dissipation. We also find that the wave frequency for a column plasma is always smaller than that for a bulk plasma due to the geometric effect of the bounded plasma. However, the growth rate for a column plasma is larger than that for a bulk plasma. In addition, the range of wave number for propagation is suppressed by the finite radius of the column. For the numerical analysis, we would 
scale the frequency, the wave number and the radius of column. If we let $\omega=\omega_{R}+i \gamma$, where $\omega_{R}$ and $\gamma$ are the real and the imaginary part of $\omega$, and scale the frequency as $\bar{\omega} \equiv \omega / \omega_{p e}$, then the imaginary part of the scaled wave, $\bar{\gamma}$, can be given as

$$
\bar{\gamma}\left(\bar{k}_{z}\right)=\frac{\bar{\omega}_{p i}^{2}}{2 \bar{v}\left(1-\bar{k}_{z}\right)}\left(1-\frac{\alpha_{0 n}^{2}}{k_{z}^{2} R^{2}}\right)^{-1 / 2},
$$

where other scaled quantities are defined by $\bar{\omega}_{p i} \equiv \omega_{p i} / \omega_{p e}, \quad \bar{v} \equiv v / \omega_{p e}, \quad \bar{k}_{z} \equiv k_{z} u_{i} / \omega_{p e}$ and $\bar{R} \equiv R \omega_{p e} / u_{i}$. Therefore, the instability of the wave occurs when the scaled axial wave number is greater than unity. However, the wave will be damped for $\alpha_{0 n} / \bar{R}<\bar{k}_{z}<1$. Hence, we have found that the unstable mode of the space-charge wave would be caused by the coupling between the electron plasma wave and the positive dissipation due to the collision-dominated streaming ions. In addition, the damping mode would be caused by the coupling between the electron plasma wave and the negative dissipation.

\section{Results and Discussions}

Figure 1 shows the scaled growth rate $\bar{\gamma}(>0)$ of the space-charge wave in a column filled with the collision-dominated streaming ions as a function of the scaled axial wave number for various values of the root of the zero-the order Bessel function. In this figure, we have found that the space-charge wave can have unstable growing modes for large axial wave numbers. As shown in this figure, the scaled growth rate of the space-charge wave increases with an increase of the order of roots of the Bessel function. However, it is found that the influence of harmonic-order on the scaled growth rate decreases with increasing scaled axial wave number. Hence, we have found that the harmonic-order effect on the scaled growth rate decreases with an increase of the streaming velocity.

Figure 2 shows the scaled growth rate $\bar{\gamma}(>0)$ of the space-charge wave as a function of the scaled axial wave number for various values the scaled radius of the plasma column. From this figure, it is found that $\bar{\gamma}$ decreases with an increase of $\bar{R}$. It is also understood that $\bar{\gamma}$ decreases with an increase of the electron plasma frequency. In addition, it is found that the influence of radial-size on $\bar{\gamma}$ decreases with increasing scaled axial wave number. Figure 3 shows the scaled damping rate $\bar{\gamma}(<0)$ of the space-charge wave as a function of the scaled axial wave number for various values the scaled radius of the plasma column. From this figure, it is found that the space-charge wave can be damped for small axial wave numbers. As it is seen, the damping domain increases with an increase of the scaled radius of the plasma column. Hence, we have found that the ion collision plays a crucial role in the physical characteristics of the space-charge wave. These result would be useful for understanding the propagation of the space-charge wave in a dusty plasma column.

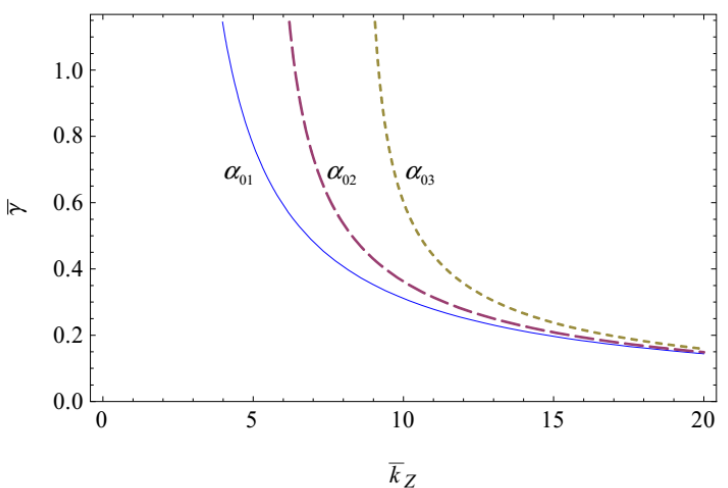

Figure 1 - The scaled growth rates $\bar{\gamma}$ are drawn as a function of the scaled wave number $\bar{k}_{z}$ for various roots of the Bessel function. The solid, dashed, and dotted lines are plotted for the roots $\alpha_{01}=2.4048, \alpha_{02}=5.5201$, and $\alpha_{03}=8.6537$, respectively, for $\bar{v}=1 \times 10^{-4}$ and $\bar{R}=1$

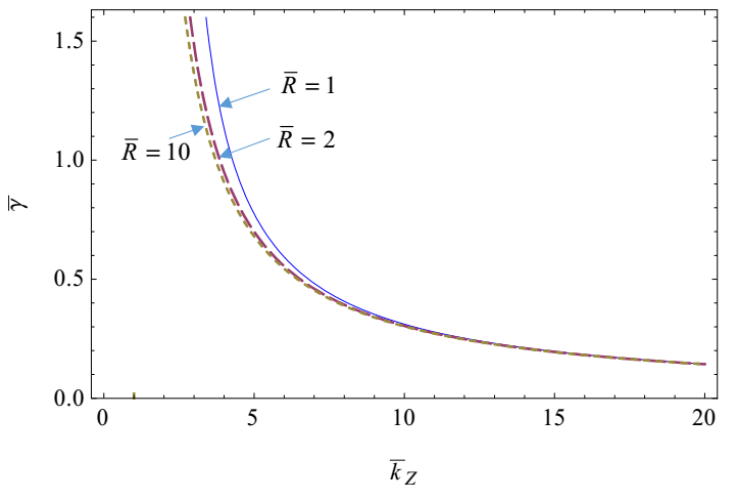

Figure 2 - The scaled growth rates $\bar{\gamma}$ are drawn as a function of the scaled wave number $\bar{k}_{z}$ for various roots of the Bessel function. The solid, dashed, and dotted lines are plotted for the roots $\bar{R}=1, \bar{R}=2$, and $\bar{R}=10$, respectively, for $\bar{v}=1 \times 10^{-4}$ and $\alpha_{01}=2.4048$. 


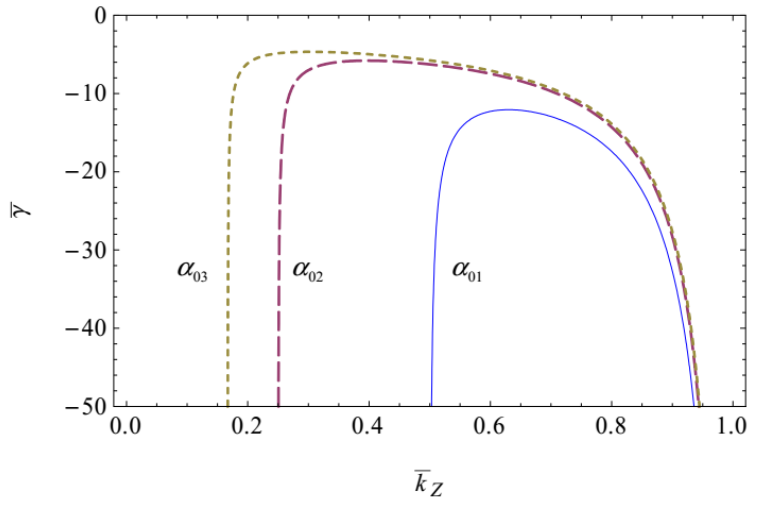

Figure 3 - The scaled damping rates $\bar{\gamma}$ are drawn as a function of the scaled wave number $\bar{k}_{z}$ for various roots of the Bessel function. The solid, dashed, and dotted lines are plotted for the roots $\alpha_{01}=2.4048, \alpha_{02}=5.5201$, and $\alpha_{03}=8.6537$, respectively, for $\bar{v}=1 \times 10^{-4}$ and $\bar{R}=1$.

\section{Conclusions}

The new results on the dispersion relation have been obtained for the space-charge wave propa- gating in a dusty plasma column with collisional streaming ions. It is has been also derived the growth rate of the space-charge wave. The geometric and collisional effects on the growth rate have been investigated. Obtained results show that the unstable mode of the space-charge wave would be caused by the coupling between the electron plasma wave and the positive dissipation due to the collision-dominated streaming ions. In addition, the damping mode would be caused by the coupling between the electron plasma wave and the negative dissipation. The space-charge wave can have unstable growing modes for large axial wave numbers. Influence of harmonic-order on the scaled growth rate decreases with increasing scaled axial wave number. And the harmonic-order effect on the scaled growth rate decreases with an increase of the streaming velocity. The space-charge wave can be damped for small axial wave numbers. Damping domain increases with an increase of the scaled radius of the plasma column. It is shown that the ion collision plays a crucial role in the physical characteristics of the space-charge wave.

\section{References}

[1] N. N. Rao, P. K. Shukla, M. Y. Yu. Dust-acoustic waves in dusty plasmas // Planet. Space Sci. - 1990. - Vol. 38. - P. 543.

[2] P. K. Shukla, V. P. Silin. Dust ion-acoustic wave //Phys. Scripta. - 1992. - Vol. 45. - P. 508.

[3] D. A. Mendis. Progress in the study of dusty plasmas // Plasma Sources Sci. Technol. - 2002. - Vol. 11. - P. A219.

[4] N. X. Wei, J. K. Xue. Bounded dust-acoustic waves in a cylindrically bounded collisional dusty plasma with dust charge variation // Phys. Plasmas. - 2006. - Vol. 13. - P. 052101.

[5] V. Yaroshenko, H. Thomas, G. E. Morfill. Dust density waves in a complex plasma layer // Phys. Plasmas. - 2007. - Vol. 14. - P. 082104.

[6] R. L. Merlino, A. Barkan, C. Thompson, N. D'Angelo. Laboratory studies of waves and instabilities in dusty plasmas // Phys. Plasmas. - 1998. - Vol. 5. - P. 1607.

[7] I. Pilch, A. Piel, T. Trottenberg, M. E. Koepke. Dynamics of small dust clouds trapped in a magnetized anodic plasma // Phys. Plasmas. - 2007. - Vol. 14. P.123704.

[8] M.-J. Lee, Y.-D. Jung. The first radial-mode Lorentzian Landau damping of dust acoustic spacecharge waves // Phys. Plasmas. - 2016. - Vol. 23. P.052105.
[9] M.-J. Lee, Y.-D. Jung. Effect of turbulence on the dissipation of the space-charge wave in a bounded turbulent plasma column // Phys. Plasmas. - 2016. Vol.23. - P. 072107.

[10] M.-J. Lee, Y.-D. Jung. Bifurcation of spacecharge wave in a plasma waveguide including the wake potential effect // Phys. Plasmas. - 2016. - Vol. 23. P.094501.

[11] M. Rosenberg, P. K. Shukla. Ion-dust streaming instability in a radially bounded dusty plasma // J. Plasma Phys. - 2011. - Vol. 77. - P. 709.

[12] O. Buneman. Instability, Turbulence, and Conductivity in Current-Carrying Plasma // Phys. Rev. Lett. - 1958. - Vol. 1. - P. 8.

[13] A. Hasegawa. Plasma Instabilities and Nonlinear Effects // Springer. Berlin. - 1975. - PP. 220.

[14] A. Hirose, H. M. Skarsgard. Nonlinear evolution of Buneman instability // Phys. Rev. Lett. - 1976. - Vol. 33. - P. 252.

[15] O. Mitarai, Y. Kawai, F. Kako. Numerical Analysis of Current-Driven Instabilities for Large Drift Velocities in a Magnetic Field // J. Phys. Soc. Japan. 1980. - Vol. 49. - P. 1974.

[16] A. Sitenko, V. Malnev. // Plasma Physics Theory. Chapman \& Hall. London. - 1995. - PP. 403. 
[17] H. L. Pécseli. // Waves and Oscillations in Plasmas. CRC Press. Boca Raton. Florida. USA. - 2013. - PP. 575.

[18] H. Kählert. Ion-dust streaming instability with non-Maxwellian ions // Phys. Plasmas. - 2015. - Vol. 22. - P. 073703

[19] M.-J. Lee, Y.-D. Jung. Influence of magnetic field and density on the streaming instability in magnetized pair plasmas // Plasma Sources Sci. Technol. - 2015. - Vol. 24. - P. 032001.

[20] V. N. Tsytovich, G. E. Morfill, S. V. Vladimirov,
H. Thomas. // Elementary Physics of Complex Plasmas. Springer. Berlin. Germany. - 2008. - PP. 370.

[21] T. S. Ramazanov, Zh. A. Moldabekov, K. N. Dzhumagulova, M. M. Muratov. Pseudopotentials of the particles interactions in complex plasmas // Phys. Plasmas. - 2011. - Vol. 18. - P. 103705.

[22] N. A. Krall, A. W. Trivelpiece. // Principles of Plasma Physics. McGraw-Hill. New York. - 1973. - PP. 704.

[23] K.-Z. Zhang, J.-K. Xue. Streaming instability in bounded three-component quantum plasmas // Phys. Plasmas. - 2010. - Vol. 17. - P. 032113. 